\title{
All-Optical Phase and Amplitude Regeneration Properties of a 40Gbit/s DPSK Black-Box Phase Sensitive Amplifier
}

\author{
F. Parmigiani ${ }^{1}$, R. Slavík ${ }^{1}$, J. Kakande ${ }^{1}$, L. Grüner-Nielsen ${ }^{2}$, D. Jakobsen ${ }^{2}$, S. Herstrøm², R. \\ Weerasuriya $^{3}$, S. Sygletos ${ }^{3}$, A. D. Ellis ${ }^{3}$, P. Petropoulos ${ }^{1}$, and D. J. Richardson ${ }^{1}$ \\ (1) Optoelectronics Research Centre, University of Southampton, Southampton, SO17 1BJ, UK, \\ frp@orc.soton.ac.uk. \\ (2) OFS, Priorparken 680, 2605 Brøndby, Denmark. \\ (3) Tyndall National Institute and Department of Physics, University College Cork, Cork, Ireland.
}

\begin{abstract}
We experimentally study the pure amplitude and phase regeneration capabilities of a blackbox degenerate four wave mixing (FWM) based bit-rate-flexible phase sensitive amplifier (PSA) for a $40 \mathrm{Gbit} / \mathrm{s}$ differential phase-shift keyed (DPSK) signal.
\end{abstract}

\section{Introduction}

Linear and nonlinear phase noise represents a significant limiting factor to data transmission using (differential) phase-shift keying (PSK) modulation formats ${ }^{1}$, which are recently emerging as alternative solutions to on-off keying (OOK), especially for long-haul transmission. Consequently, there is great interest in developing all-optical regeneration schemes capable of eliminating phase (and ideally also amplitude) noise for (D)PSK signals. To date most work on all-optical regeneration of DPSK signals has focused on the elimination of amplitude noise only, which can be achieved using cross-phase modulation (XPM) in a nonlinear amplifying loop mirror ${ }^{2}$ or using saturation of FWM interactions caused by pump depletion in a nonlinear fibre ${ }^{3-4}$. A different approach to the elimination of amplitude and phase noise is to adopt some form of phase-toamplitude format conversion and then to apply amplitude regeneration of the amplitude encoded signal ${ }^{5}$. However, direct elimination of the phase noise would be a far more desirable and simpler solution.

Phase and amplitude regeneration can be achieved directly by exploiting the phase squeezing capability and the saturated operating regime of phase-sensitive amplifiers respectively. Initial proof of concept results have recently been demonstrated by Croussore et al. $^{6-8}$ in either single- or dual-pump configurations using either interferometric configurations based on nonlinear optical loop mirrors $^{6}$, or straight line fibre-based FWM processes $^{7-8}$. However, to establish a suitable phase relationship between the pump(s) and the signal, direct access to the original signal carrier (i.e., the continuous wave (CW) laser prior the modulator) was essential for the straight line FWM-based $\mathrm{PSA}^{7,8}$, making the regenerator impractical for use in a real transmission system where carrier recovery and phase-locking between the newly generated pump(s) needs to be obtained from the incoming DPSK signal itself. We have recently proposed and experimentally demonstrated a scheme that mitigates this issue allowing for the realization of a black-box regenerator operated in a dualpump degenerate PSA configuration ${ }^{9}$. While this scheme was shown to provide impressive phase regeneration capabilities at $10 \mathrm{Gbit} / \mathrm{s}$ and 40Gbit/s the amplitude regeneration capabilities of this scheme were not yet investigated.

Here, we demonstrate the amplitude noise regeneration capabilities for the FWM-based PSA of a 40Gbit/s DPSK signal and further investigate the performance limitations for both phase and amplitude regeneration.

\section{Experimental Set-up and Results}

The experimental setup is shown in Fig. 1. The data signal is a 40Gbit/s non-return-to-zero (NRZ)-DPSK, $2^{31}-1$ pseudo-random bit sequence (PRBS). In order to emulate the effects of phase and amplitude noise, the signal phase or amplitude was modulated in a

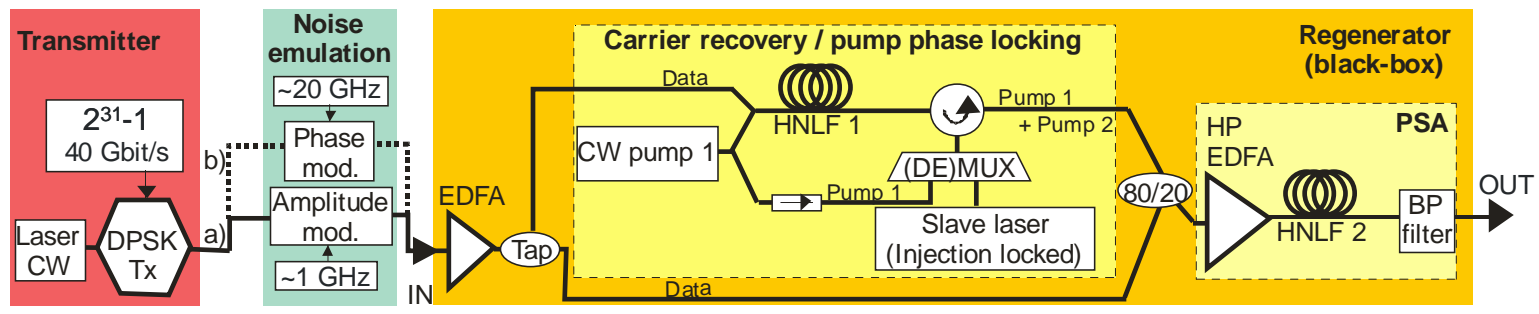

Fig. 1: Experimental set-up of the 40Gbit/s phase and amplitude regenerator. 
deterministic fashion using either of two additional phase and amplitude modulators, driven sinusoidally at frequencies of $\sim 20 \mathrm{GHz}$ and $\sim 1 \mathrm{GHz}$, respectively. This way of adding noise allowed us to independently emulate different levels of phase and amplitude noise in order to carry out a comprehensive study of the regenerator performance.

The distorted signal was then launched into the black-box regenerator. Part of the signal was tapped-off first to enable the frequency/phase locking of the two local pumps used in the degenerate PSA. The tapped-signal and a narrow linewidth $\mathrm{CW}$ laser (Pump 1) detuned from the signal by $200 \mathrm{GHz}$ were first combined in a germano-silicate highly nonlinear fibre (HNLF) (length, dispersion, nonlinear coefficient and attenuation of $500 \mathrm{~m},-0.09 \mathrm{ps} / \mathrm{nm} / \mathrm{km}$, $11.5 \mathrm{~W}^{-1} \mathrm{~km}^{-1}$ and $0.83 \mathrm{~dB} / \mathrm{km}$, respectively) to parametrically generate an idler wave that served as the seed for the second pump. Note that due to the phase erasure process, the binary data modulation is not transferred to the idler $^{9,10}$. Then, the weak idler wave was filtered and injected into a (slave) semiconductor laser to generate the second phase-locked pump (Pump 2) by means of a multiplexer ${ }^{11}$. As the injection locking is a much slower process than FWM, any high frequency phase fluctuations (e.g., bit-to-bit phase variations) present on the original data signal were not transferred onto the output of the slave laser and amplitude noise is substantially suppressed. Pump 1 and Pump 2 were then coupled together with the data signal, amplified to a total power of $\sim 34 \mathrm{dBm}$ and launched into a PSA based on an aluminosilicate HNLF with strain controllably applied along its length to increase the Stimulated Brillouin Scattering (SBS) threshold (alleviating the need for any active SBS suppression scheme). The length, dispersion, polarization mode dispersion, nonlinear coefficient and attenuation of this fibre are $177 \mathrm{~m}$, $-0.13 \mathrm{ps} / \mathrm{nm} / \mathrm{km}, \quad 0.11 \mathrm{pskm}^{-0.5}, \quad 7.1 \mathrm{~W}^{-1} \mathrm{~km}^{-1}$ and $15 \mathrm{~dB} / \mathrm{km}$, respectively. The relative powers of the pumps and signal were adjusted for optimal regeneration performance. Any slow (sub kHz) relative phase drifts between the interacting waves picked up due to acoustic and thermal effects present prior to the PSA fibre were compensated for by an electrical phase-locked loop that controlled a piezoelectric-based fibre stretcher in the pump path (to simplify the schematic, this feedback system is not shown in Fig.1).

Fig.2 shows the corresponding optical spectra at the PSA HNLF output (before filtering out the pumps) for maximum and minimum gain,

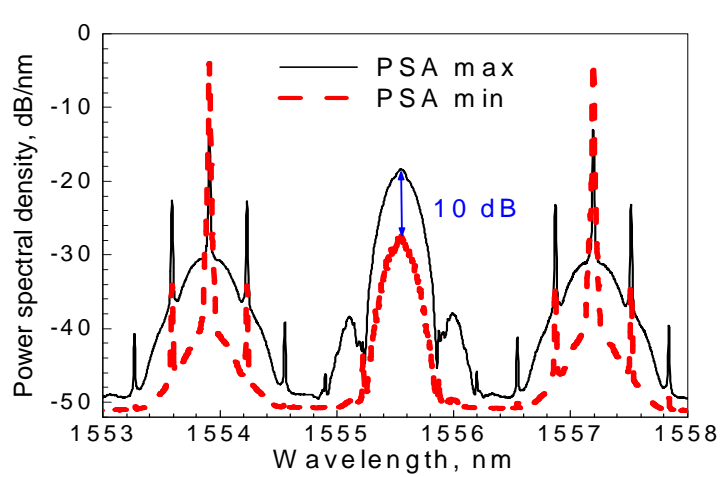

Fig. 2: Optical spectrum at the output of the PSA for maximum and minimum amplification.

showing a signal total swing of $\sim 10 \mathrm{~dB}$. Note that a similar swing can be seen for the depleted pumps.

The performance of the regenerator was first studied when different levels of deterministic phase-only noise were added to the signal using eye diagrams and by measuring bit error ratio (BER) curves. Following demodulation in a 1-bit delay line interferometer (DLI) and balanced detection using a dual port optical sampling oscilloscope, the eyes measured at the input and output of the regenerator for no noise and for the highest level of the added phase noise are shown in the inset of Fig. 3, highlighting that the regenerator, which acts on the absolute bit phase, is capable of restoring a completely closed differentially detected eye. To provide a more quantitative analysis of its performance, BER curves are shown in Fig. 3. Note that in this case single-receiver detection (after the DLI) was used. At the input of the regenerator, the added phase noise caused error flooring in the BER curves from $10^{-6}$ to $10^{-3}$. At the output of the regenerator error-free performance is achieved for all the noise levels with power penalties below $3.5 \mathrm{~dB}$ as compared to the backto-back case. For the highest level of noise (corresponding to a completely closed eye at the input of the regenerator), the regenerator output eye diagrams are still wide open, however the corresponding BER curve starts to show a slight error floor due to phase to amplitude noise, illustrating the PSA limitations.

The performance of the regenerator was then studied when different levels of amplitude-only deterministic noise were added to the signal. Note that the same experimental conditions (signal/pumps powers) were preserved at the input of the PSA in this case. The demodulated eyes for no noise and for the highest level of the added amplitude noise (corresponding to amplitude variations of $\pm 50 \%$ ) are shown in the inset of Fig. 4, highlighting the good amplitude regeneration capability. Again, to allow a more 


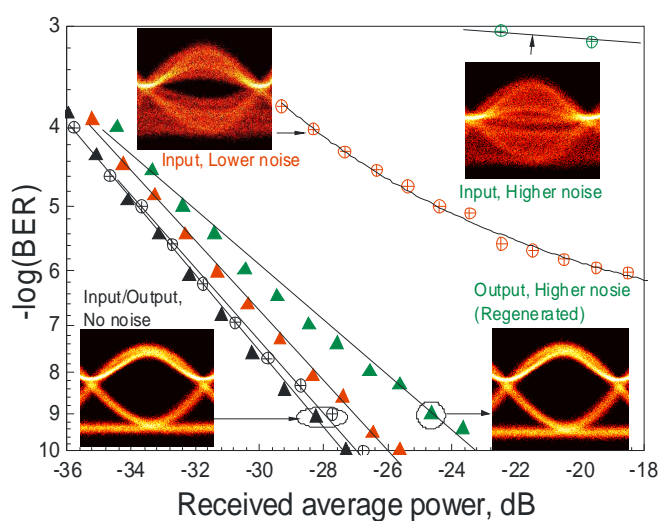

Fig. 3 : BER curves at the input (circles) and output (triangles) of the regenerator for different values of added phase noise (single-receiver detection) and corresponding demodulated eye diagrams after balanced detection.

quantitative analysis of the performance BER curves are shown in Fig. 4. The use of balanced detection should give a $3 \mathrm{~dB}$ overall sensitivity improvement as compared to single-receiver detection (irrespective of the addition or not of phase noise). However, balanced detection should provide significant additional resilience to amplitude noise relative to single-receiver detection since variations in power are cancelled to first order.

At the input of the regenerator, the added amplitude noise caused the BER curves to reach levels ranging from $10^{-6}$ to $10^{-4}$ for the maximum input power available into the receiver $(-22 \mathrm{dBm})$. At the output of the regenerator errorfree performance is achieved for all noise levels investigated with up to $3 \mathrm{~dB}$ power penalties as compared to the PSA output with no input signal degradation. For the highest level of noise (corresponding to an almost closed eye at the input of the regenerator), the regenerator output eye diagrams are again wide open, although the corresponding BER curve starts to show a modest deviation from a straight line resulting from the limitations of the PSA.

Finally, preliminary studies, not reported here, suggest the suitability of the PSA scheme for simultaneous regeneration of both amplitude and phase noise.

\section{Conclusions}

We have experimentally demonstrated the phase and amplitude regeneration of a blackbox 40Gbit/s DPSK PSA using degenerate FWM in a high SBS-threshold highly nonlinear fibre. The limitations of the system for extreme cases of amplitude and phase variations (corresponding to completely closed eyes before the regenerator) are also reported and

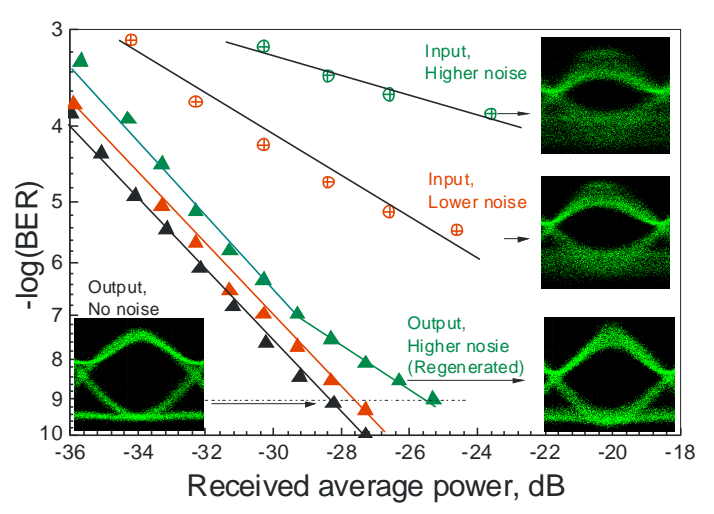

Fig. 4: BER curves at the input (circles) and output (triangles) of the regenerator for two values of added amplitude noise corresponding to $\pm 30 \%$ ('Lower noise') and $\pm 50 \%$ ('Higher noise') variations in the peak power (single-receiver detection) and corresponding demodulated eye diagrams after balanced detection.

discussed.

\section{Acknowledgments}

The authors would like to thank Eblana Photonics Inc., Dublin, Ireland, for providing the coaxial discrete mode laser used in the experiment and $\mathrm{Dr}$ Adonis Bogris for fruitful discussions. This research has received funding from the European Communities Seventh Framework Programme FP/2007-2013 under grant agreement 224547 (PHASORS) and by Science Foundation Ireland under grant number 06/IN/I969.

\section{References}

1 J. P. Gordon, et al., Opt. Lett. 15 (23), 13511353 (1990).

2 K. Cvecek, et al., Opt. Express 16, 1923-1928 (2008).

3 K. Croussore, et al., Electron. Lett., 43 (3), 177 - 178, (2007).

4 M. Matsumoto et al., Opt. Express 15, 80948103 (2007).

5 M. Matsumoto et al., Opt. Express 16, 1116911175 (2008).

$6 \mathrm{~K}$. Croussore, et al., Opt. Express, 14, 20852094 (2006).

$7 \mathrm{~K}$. Croussore et al., IEEE/LEOS Winter Topical Meeting Series, MB1.3, (2008).

$8 \mathrm{~K}$. Croussore et al., IEEE Phot. Technol. Lett., 19 (11), 864-866, (2007).

9 Parmigiani F. et al. Proc. OFC'10, PDPC3 (2010).

10 G.-W. Lu, et. al., IEEE Phot. Technol. Lett. 21, 322-324 (2009).

11 R. Weerasuriya, et al., Proc. OFC'10, OWT6 (2010). 